\title{
Recent Advances in SPECT Imaging
}

\author{
Mark T. Madsen \\ Department of Radiology, University of Iowa, Iowa City, Iowa
}

SPECT is a rapidly changing field, and the past several years have produced new developments in both hardware technology and image-processing algorithms. At the component level there have been improvements in scintillators and photon transducers as well as a greater availability of semiconductor technology. These devices permit the fabrication of smaller and more compact systems that can be customized for particular applications. New clinical devices include high-count sensitivity cardiac SPECT systems that do not use conventional collimation and the introduction of diagnostic-quality hybrid SPECT/CT systems. While there has been steady progress with reconstruction algorithms, exciting new processing algorithms have become commercially available that promise to provide substantial reductions in SPECT acquisition time without sacrificing diagnostic quality. Preclinical small-animal SPECT systems have become a major focus in nuclear medicine. These systems have pushed the limits of SPECT into the submillimeter range, making them valuable molecular imaging tools capable of providing information unavailable from other modalities.

Key Words: SPECT; scintillators; photon transducers; semiconductors; cardiac SPECT; SPECT/CT; image processing; smallanimal SPECT

J Nucl Med 2007; 48:661-673

DOI: 10.2967/jnumed.106.032680

\section{$\mathbf{F}$} rom the beginning of radionuclide imaging, there has been a dedicated effort to produce tomographic images of the internal distribution of radiopharmaceuticals. Although the early development of SPECT will not be discussed in this article, an excellent review of the key investigators and their ground-breaking work can be found in a recent article by Jaszczak (1). The present article will focus on recent developments in SPECT that cover approximately the past $3 \mathrm{y}$. I will begin by briefly reviewing the fundamental physical assumptions underlying SPECT and follow that with a discussion of the advances in detection instrumentation. Clinical and research devices designed for patient studies will be reviewed next. Small-animal SPECT systems will also be described. These sections will be followed by a

\footnotetext{
Received Oct. 5, 2006; revision accepted Jan. 31, 2007.

For correspondence or reprints contact: Mark T. Madsen, PhD, Department of Radiology, University of lowa, 200 Hawkins Dr., lowa City, lowa 52242.

E-mail: Mark-madsen@uiowa.edu

COPYRIGHT @ 2007 by the Society of Nuclear Medicine, Inc.
}

review of reconstruction algorithms and correction methods. An outstanding in-depth source of information on all of these topics for the interested reader can be found in the book Emission Tomography: The Fundamentals of PET and $\operatorname{SPECT}(2)$.

Conventional nuclear medicine images compress the 3-dimensional (3D) distributions of radiotracers into a 2-dimensional image. As a result, the contrast between areas of interest and the surrounding territory is often significantly reduced. This reduction limits the diagnostic information that is available in the study. In addition, the exact location of an abnormality can be difficult to determine. Tomographic images remove these difficulties but at the price of longer acquisition times, poorer spatial resolution, and the susceptibility to artifacts. Recent advances in SPECT instrumentation and processing have made marked improvements in each of these areas.

\section{SPECT FUNDAMENTALS}

The fundamental objective of any tomographic imaging is to determine the internal distribution of an object solely from external measurements. This can be accomplished only if the following requirements are met: (a) a complete set of projections is acquired, (b) the internal distribution is not spatially or temporally changing during the time that the projections are acquired, (c) the detectors that acquire the projections have uniform detection sensitivity that remains constant throughout the acquisition, and (d) the center of rotation is accurately known. (The center of rotation is a coordinate that defines the common center of the acquired projections, and its accurate location is required for proper alignment of the projections during reconstruction.) If measurements can be made that meet these criteria, then an accurate reconstruction of the internal distribution can be determined.

It is important to note that the information collected by SPECT systems (and also for PET) does not meet the definition of a projection. The $\gamma$-ray emissions collected by the SPECT systems are not linearly related to the ray sums of activity in the patient because of tissue attenuation. As a consequence, if these attenuated projections are reconstructed, the resulting tomographic slices contain artifacts and will not accurately reflect the true internal distribution. Factors other than attenuation limit the quality of reconstructed images. These include spatial resolution, scattered 
radiation, and statistical fluctuations (image noise). The advances in SPECT are directly tied to improving the quality and accuracy of the acquired projections either through better instrumentation or better correction algorithms.

\section{DETECTION INSTRUMENTATION}

The projection information required for SPECT is acquired by $\gamma$-ray detectors, and much of the quality of the projection depends on the properties of these detectors. We desire detectors with the following characteristics: (a) high intrinsic efficiency, (b) good energy resolution, and (c) good intrinsic spatial resolution. Materials with high atomic numbers and high density are important for detector efficiency. Both energy resolution and spatial resolution depend on the size of the signal generated with each detected event. Scintillation cameras using $\mathrm{NaI}(\mathrm{Tl})$ have high intrinsic efficiency for $\gamma$-rays of $<200 \mathrm{keV}$, but the energy resolution and intrinsic spatial resolution are only moderate. As a result, there has been an ongoing search for better detectors as well as better detector configurations. Efforts have also been made to make smaller and more compact imaging systems for imaging small organs and for small-animal imaging (3-5).

\section{Scintillators}

Materials that emit visible light when they absorb energetic charged particles, $\gamma$-rays, or x-rays are called scintillators $(6,7)$. Table 1 lists the physical properties of the materials that will be discussed in this section. Scintillators have several advantages as $\gamma$-ray detectors, including high intrinsic efficiency and moderate cost. They can also be fabricated into a large variety of shapes and sizes. However, scintillators require an additional stage to convert the scintillation light into an electronic pulse. Light is lost at the interface between the detector and the photon transducer, which limits energy resolution.

The materials shown in Table 1 have comparable effective atomic numbers and densities, which mean they will have similar photopeak efficiencies. The persistence of the scintillation limits the counting rate capability of the detectors, although counting rate losses are rarely a problem with SPECT when conventional collimation is used. The wavelength of the scintillation determines what kind of photon transducer can be used to turn the scintillation into an electronic pulse. The brightness of the scintillation expressed in terms of the output relative to $\mathrm{NaI}(\mathrm{Tl})$ directly affects the potential energy resolution.

Along with the trend for making smaller and more compact imaging systems, new $\gamma$-camera designs have featured pixelated detectors, where the field of view is covered by an array of individual scintillators with a face size of typically 2-3 mm instead of one large crystal, as with a conventional Anger scintillation camera (Fig. 1A). Each approach has its own advantages. The large detector geometry of a conventional scintillation camera is less costly to assemble and provides continuous sampling. Position information is determined by Anger logic or similar weighted averaging of photomultiplier tube (PMT) signals from an array of PMTs covering the detector back surface. One disadvantage to this approach is that the Anger logic breaks down near the detector edge, resulting in several centimeters of "dead" space. This is not much of a concern with large detectors but becomes substantial for smaller field-of-view systems. Pixelated detectors do not have dead edges and are preferred for small-field-of-view configurations. The sampling and intrinsic spatial resolution of pixelated systems are determined by the face size of the individual detector elements. In addition to the increased assembly costs, energy resolution is usually worse for the pixelated detectors because of reduced light transmission and subsequent collection by the photon transducers $(3,5,8)$.

Thallium-activated sodium iodide, $\mathrm{NaI}(\mathrm{Tl})$ is still the most commonly used scintillator for SPECT applications. In most clinical SPECT systems, the $\mathrm{NaI}(\mathrm{Tl})$ detector is one large crystal as described above. However, $\mathrm{NaI}(\mathrm{Tl})$ can be pixelated and several small-field-of-view devices, including small-animal SPECT systems, are configured that way. For clinical SPECT, the NaI(Tl) thickness is typically $6-10 \mathrm{~mm}$. It is possible to have $\gamma$-camera detectors made with $\mathrm{NaI}(\mathrm{Tl})$ crystals that are 25.4-mm thick (StarBrite; Saint-Gobain Crystals and Detectors) to obtain increased efficiency for high-energy $\gamma$-emitters (9). As shown in Figure 1B, grooves are machined into the top surface of the detector to prevent light diffusion and to maintain acceptable intrinsic spatial resolution (4.1 $\mathrm{mm}$ at $140 \mathrm{keV})$.

Thallium-activated cesium iodide competes well with $\mathrm{NaI}(\mathrm{Tl})$ in terms of efficiency and it is not strongly hygroscopic. It has a significantly longer scintillation time, leading to a larger dead time, but that is rarely a concern for SPECT. However, the scintillation light from CsI(Tl) has a

TABLE 1

Scintillators

\begin{tabular}{lccccc}
\hline Scintillator & $\begin{array}{c}\text { Atomic number } \\
\text { Z effective }\end{array}$ & Density $\rho\left(\mathrm{g} / \mathrm{cm}^{3}\right)$ & Decay time $\tau(\mathrm{ns})$ & Wavelength $\lambda(\mathrm{nm})$ & $\begin{array}{c}\text { Relative light output } \\
(\% \text { of Nal}(\mathrm{Tl}))\end{array}$ \\
\hline $\mathrm{Nal}(\mathrm{Tl})$ & 50 & 3.67 & 200 & 415 & 100 \\
$\mathrm{Csl}(\mathrm{TI})$ & 54 & 4.5 & 1,000 & 550 & $45\left(118^{\star}\right)$ \\
$\mathrm{Csl}(\mathrm{Na})$ & 54 & 4.51 & 630 & 420 & 85 \\
$\mathrm{LaBr}_{3}: \mathrm{Ce}$ & 47 & 5.3 & 25 & 360 & 160
\end{tabular}

${ }^{*}$ Represents total light output. Because of the long wavelength of the scintillation, the effective signal for $\mathrm{Csl}(\mathrm{TI})$ is only $45 \%$ of $\mathrm{Nal}(\mathrm{TI})$. 


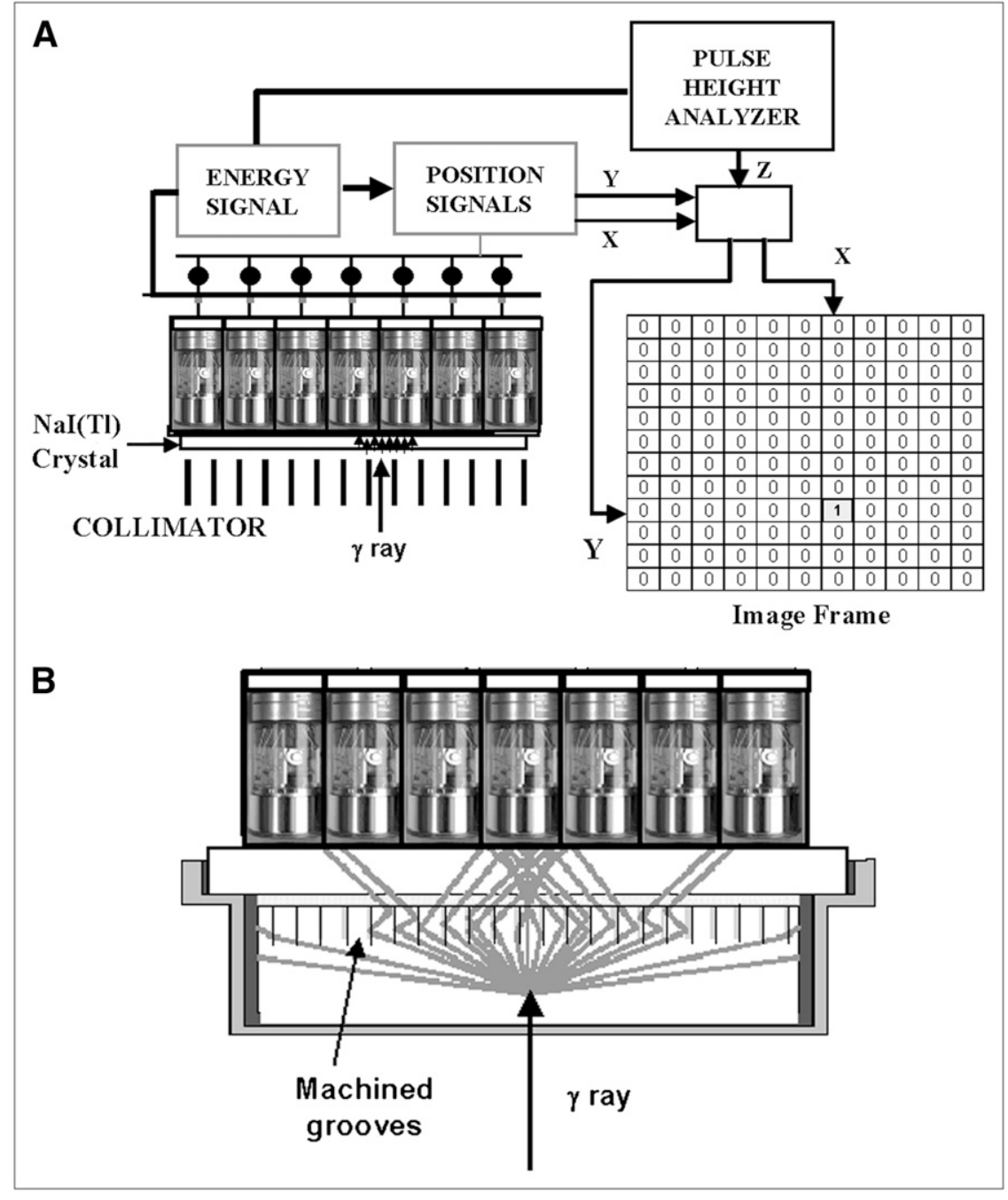

FIGURE 1. Scintillation camera. (A) In a conventional scintillation camera, light from the scintillation is sampled by an array of photomultipler tubes, which generate an energy signal as well as an $x, y-$ coordinate pair. If the event falls within the pulse-height analyzer energy window, an image matrix element is incremented at the associated $x, y$-location. (B) With StarBrite technology (Saint-Gobain Crystals and Detectors), grooves are machined into the back surface of the 1-in. thick $\mathrm{Nal}(\mathrm{TI})$ crystal to limit the spread of the scintillation light, resulting in improved intrinsic spatial resolution. longer wavelength that is not as well matched to PMTs as sodium iodide $(6,7)$. As a result, its performance with PMTs is worse than $\mathrm{NaI}(\mathrm{Tl})$ even though the total number of scintillation photons (including the infrared) is actually about $18 \%$ higher than $\mathrm{NaI}(\mathrm{Tl})$. However, these photons are detected with a high quantum efficiency with photodiodes that can replace PMTs. This approach is used by Digirad in their Cardius product. Sodium-activated cesium iodide, $\mathrm{CsI}(\mathrm{Na})$, is similar to $\mathrm{CsI}(\mathrm{Tl})$, but with an emission that is better matched to PMTs. It is currently being used as the detector in the LinoView small-animal SPECT system (10).

Lanthanum bromide, $\mathrm{LaBr}_{3}$, is a scintillation that has garnered attention as a potential detector for time-of-flight PET, but its properties are also attractive for single-photon imaging $(6,11)$. $\mathrm{LaBr}_{3}$ has a very high light output with a correspondingly improved energy resolution $(<6 \%$ at $140 \mathrm{keV})$ and an intrinsic efficiency comparable to $\mathrm{NaI}(\mathrm{Tl})$. In addition, $\mathrm{LaBr}_{3}$ is a very fast detector and potentially very useful for approaches such as Compton $\gamma$-cameras, where high counting rates are likely to be encountered. It is also being used as the detector in a recently reported small-animal SPECT system (12).

\section{Photon Transducers}

PMTs have been used for $>40$ y to convert scintillation light into electronic signals (Fig. 2A). PMTs have very large electronic gains $\left(10^{6}\right)$ with relatively low noise. However, their quantum conversion efficiency is low ( $\sim 20 \%)$, leading to a significant loss of signal that affects both energy resolution and intrinsic spatial resolution. It is difficult to maintain the long-term stability of PMTs because they are susceptible to environmental influences such as temperature, humidity and magnetic fields and their properties change as they age. In addition, they are bulky as well as expensive. Improved replacements for conventional PMTs are available and have been incorporated into some commercial $\gamma$-ray imaging systems (13). These devices include the positionsensitive PMT (PSPMT) and the avalanche photodiode (APD) (Figs. 2B and 2C). Other devices such as the positionsensitive APD and the silicon photomultiplier (Fig. 2D) have been recently introduced and are likely to be incorporated into SPECT instrumentation in the near future.

Conventional PMTs have no intrinsic localization-that is, there is no way of knowing what portion of the 
A
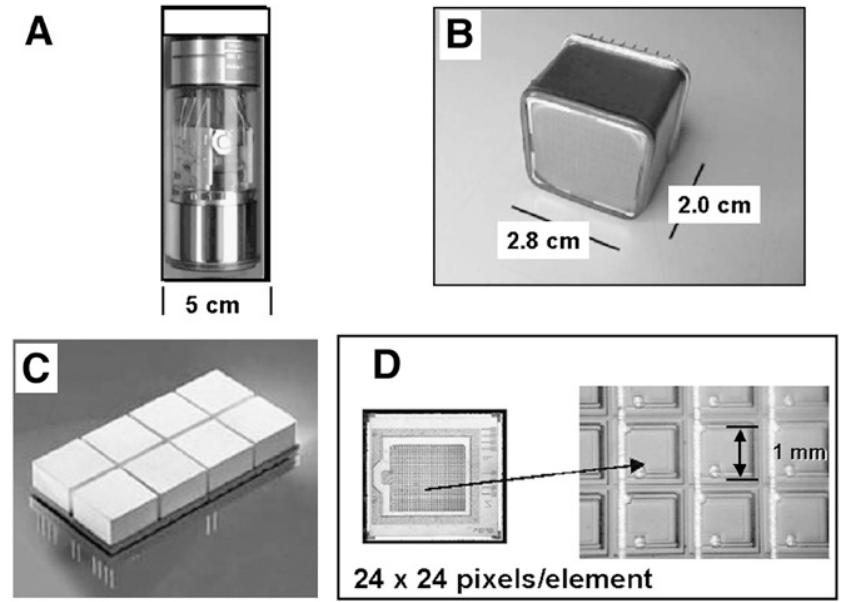

FIGURE 2. Photon transducers for converting scintillations into an electronic pulse. (A) PMT. (B) Position-sensitive PMT. (C) Avalanche photodiode. (D) Silicon photomultiplier.

photocathode interacts with the scintillations. PSPMTs are essentially a matrix of PMT elements that combine highgain capabilities with the ability to localize events to several millimeters (Fig. 2B). PSPMTs have been primarily used in small-field-of-view devices with individual, pixilated detectors (14-16). In addition to their position sensitivity and high gain, PSPMTs are more compact and more efficiently sample the detector surface than individual PMTs. However, there are wide gain variations across the field, which makes this device better suited for pixelated detectors. PSPMTs are also influenced by magnetic fields and other environmental factors (13).

APDs are solid-state photon converters (Fig. 2C). They can be conceptualized as a light-sensitive diode with a very high reverse bias (13). Incoming photons liberate charge carriers (electrons and holes) in the cathode, which are accelerated through the diode depletion zone with enough energy to create additional charge carriers. The final electronic signal is proportional to the initial number of light photons that were detected. APDs have several advantages over PMTs. They are more rugged, very compact, and largely immune to environmental factors such as magnetic fields. They operate at a lower voltage than PMTs and have a much higher quantum conversion efficiency. However, the maximum gain of currently available APDs is about 250 (compared with $10^{6}$ for PMTs). A large gain is desirable for separating the scintillation signal from noise, which is an important factor in energy resolution. APDs are especially well suited for scintillators that emit light with longer wavelengths, such as $\mathrm{CsI}(\mathrm{Tl})$, and are best suited for pixelated detectors.

The spatial resolution is limited in a conventional APD because of the channel size. This becomes a problem when the size of the scintillation crystal is smaller than the channel size. A position-sensitive APD (PSAPD) has been developed that uses charge sharing between additional electrodes on the back surface of the APD to improve event localization to approximately $0.5 \mathrm{~mm}$, and PSAPDs have been investigated for use in high-resolution small-animal SPECT systems (17). Because the dark current from this device is a significant fraction of the signal noise, the PSAPD are usually cooled to liquid nitrogen temperatures.

Another solid-state device for photon conversion that shows promise is the silicon photomultiplier (Fig. 2D). Photodiodes are analogous to ionization chambers except that the charge carriers can be liberated with significantly lower energy. The APD operates much like a proportional counter as the output signal is proportional to the initial number of photons. Within this proportional region, only the electrons have enough energy to cause an avalanche. If the bias voltage is increased to this configuration, this proportionality is lost because the holes also contribute avalanches, leading to discharge. This is referred to as the Geiger region. As in gas ionization chambers, the Geiger region is associated with a total discharge of the device regardless of how many charge carriers were initially detected. A device with a single Geiger region would not have much value as a photon detector, but in the silicon PMT, a large array of Geiger regions is closely packed together. Current devices are available with a $24 \times 24$ array of Geiger photodiodes packaged into a $1 \times 1 \mathrm{~mm}$ area. At low light levels (as from a scintillation) there is little overlapping of the photons into these regions. As a result, the integrated signal from the array is reasonably proportional to the initial light signal. Silicon photomultipliers have all of the advantages of the APDs (compactness, low sensitivity to environmental factors, high quantum efficiency) with the added advantage of very high gains $\left(10^{5}-10^{6}\right)$ at relatively low bias levels. These devices have been investigated in PET systems and are likely to find their way into scintillation-based SPECT systems as they develop (18).

\section{Semiconductors}

Semiconductor detectors are solid-state devices that provide direct conversion of absorbed $\gamma$-ray energy into an electronic signal (19). Because there is no need for an intermediate high-gain amplification stage, these devices are compact and operate at low voltage. The absorbed energy from a $\gamma$-ray interaction liberates charge carriers (electrons and holes) within the charge-free depletion zone of the semiconductor. The induced charge on the terminals generates an electronic pulse with an amplitude proportional to the absorbed energy. Because there is no intermediate conversion stage, the precision of the signal is better than that of scintillators resulting in correspondingly better energy resolution. Whereas some semiconductor detectors such as high- purity germanium (HPGe) need to be cooled to low temperatures, others such as cadmium telluride (CdTe) and cadmium zinc telluride (CZT) operate at room temperature. The primary disadvantages of semiconductor detectors have been intrinsic efficiency, especially for highenergy $\gamma$-rays, and fabrication costs. However, the technology has improved and the production costs have dropped significantly in the past decade. 
CdTe and CZT are room temperature semiconductor detectors with similar properties that have been in use for several decades (20). However, it is only in the past $5 \mathrm{y}$ that this material has been used in commercial nuclear medicine imaging devices. The intrinsic efficiency for CZT is comparable to $\mathrm{NaI}(\mathrm{Tl})$ at similar detector thickness, and energy resolution in the range of $2 \%-5 \%$ can be achieved for 140 $\mathrm{keV} \gamma$-rays. CdTe and CZT are available as pixelated detector arrays with a typical intrinsic spatial resolution of $2.4 \mathrm{~mm}$, although much finer resolution can be achieved (21). Because of the fabrication costs, these semiconductors have been used in devices with a small field of view. Example applications include the D-SPECT cardiac SPECT system and several small-animal SPECT systems.

Another position-sensitive semiconductor with very high spatial resolution is the silicon strip detector. Because of its low atomic number $(Z=14)$ and density $\left(2.3 \mathrm{~g} / \mathrm{cm}^{3}\right)$, silicon is not useful for conventional $\gamma$-ray imaging with most medium- to high-energy $\gamma$-rays. However, it is adequate for very low-energy $\gamma$-ray and x-ray emitters, such as ${ }^{125} \mathrm{I}$, and silicon strip detectors are available with pixel sizes of $<100$ $\mu \mathrm{m}$ for ultrahigh-resolution applications. Another application for which silicon strip detectors show promise is Compton $\gamma$-cameras, where both good spatial resolution and a high likelihood for Compton interactions are very desirable (22).

Another device with very high intrinsic spatial resolution is the charge-coupled device (CCD). CCDs have been used for directly detecting $\gamma$-rays from ${ }^{125} \mathrm{I}$ (23). However, like the silicon strip detector, its intrinsic efficiency is too low for most $\gamma$-rays but it can be used for energies below 30 $\mathrm{keV}$. It has also been investigated as the photon converter component for a columnar CsI(Tl) detector (24).

\section{CLINICAL DEVICES}

Most clinical SPECT systems used to perform patient studies still use scintillation cameras with $\mathrm{NaI}(\mathrm{Tl})$ used as the detector. These systems consist of one or more scintillation camera heads attached to a gantry that revolves around the patient to collect projection views. The most common configuration has 2 scintillation cameras that are either fixed at $90^{\circ}$ or $180^{\circ}$ or have the capability to be positioned at selected orientations. The typical imaging performance of this type of system is summarized in Table 2 .

\section{Radionuclide Transmission for Attenuation Correction}

Recognizing the need for correcting attenuation in areas such as the thorax, most of the SPECT manufacturers have options for performing transmission scans through the patient using an external radionuclide source with the $\gamma$-camera as the detector $(25,26)$. This converts the SPECT system into a crude CT scanner. Each manufacturer has approached this issue with a different solution but, in each case, transmission projection images are acquired at each angle of the emission scan. When the energy of the transmission source is less than that of the $\gamma$-ray associated
TABLE 2

Typical Performance Values for a Conventional SPECT System

$\begin{array}{ll}\text { No. of detector heads } & 2 \\ \text { Field of view } & 40 \times 55 \mathrm{~cm} \\ \text { Energy resolution } & 9.50 \% \\ \text { Intrinsic spatial resolution } & 3.8 \mathrm{~mm}(\mathrm{FWHM}) \\ \text { Planar count sensitivity (LEHR) } & 190 \mathrm{cps} / \mathrm{MBq} \\ & \begin{array}{l}(95 \mathrm{cps} / \mathrm{MBq} \text { per head) } \\ 10.5 \mathrm{~mm} \text { (FWHM) }\end{array} \\ \text { SPECT spatial resolution (LEHR) } & \\ & \\ \text { LEHR = low energy, high resolution; FWHM = full width at half } \\ \text { maximum; cps = counts per second. }\end{array}$

with the radiotracer (e.g., ${ }^{153} \mathrm{Gd}$ with a $100-\mathrm{keV}$ photon and ${ }^{99 \mathrm{~m} T c}$ with a $140-\mathrm{keV}$ photon), the transmission and emission counts are acquired simultaneously along with additional energy windows to estimate cross-talk between the 2 photons. The transmission information acquired in this way is very noisy because of the low count sensitivity of the $\gamma$-camera and the limited activity in the transmission source. Although the transmission noise is not as large a problem with SPECT attenuation correction as it is with PET, betterquality transmission images are desirable and are a motivation for SPECT/CT.

The initial introduction of attenuation correction produced problems with over compensation particularly in the inferior wall of the myocardium. Scattered radiation from radioactivity distributed in the liver and other tissues results in a falsely increased flux of photons from the inferior portion of the heart. Attenuation compensation amplifies this effect, which may result in an apparent perfusion defect in the anterior wall. This problem has been reduced, although not entirely eliminated, with the addition of scatter correction, primarily achieved by subtracting simultaneously acquired image data in additional off-peak energy windows that sample the scattered radiation (27).

\section{Cardiac SPECT Devices}

It is not surprising that many of the advances in SPECT instrumentation have been directed toward myocardial perfusion studies, as these are by far the most commonly performed SPECT procedures, easily exceeding the total of all other SPECT studies combined. The major commercial vendors each offer SPECT systems that are optimized for myocardial perfusion imaging. Most of the customization has been to minimize the size of the system so that it can easily fit into an office-size room. The systems offered by GE Healthcare, Philips, and Siemens are all based on conventional dual-detector $\gamma$-camera systems that revolve about the patient to collect projection information. The Cardius system from Digirad is available with up to 3 detector heads that feature pixelated $\mathrm{CsI}(\mathrm{Tl})$ crystals with APD arrays for determining event location. In this design, the detectors remain stationary and the projections are 
collected as the patient, sitting upright in a chair, is rotated. This also results in a very compact imaging system.

Several new systems have been showcased recently that are departures from the conventional SPECT system design. Although achieved in different ways, these systems provide significantly higher count sensitivity than the conventional myocardial SPECT systems so that myocardial perfusion studies can be performed faster to improve throughput and patient comfort. As shown in Figure 3A, the CardiArc has a $180^{\circ}$ arc of pixelated CZT detectors with a series of lead plates (slats) to provide axial collimation for the detectors. A curved lead plate with a set of slits is located in front of the slats. The combination of the slits and slats provides pinhole sampling similar to the SPRINT II and provides the relatively high count sensitivity achieved by this device (1). Motion of the slit plate provides all of the required angular sampling. The patient sits upright in a chair and is able to lean on the detector gantry as the motion of the plate is internal and is hidden from the patient. This design is reported to have higher count sensitivity and better spatial resolution than a conventional SPECT system. A similar slit-slat design is used in the MarC-SPECT, an investigational cardiac SPECT device in which the patient rotates while the detector and the apertures remain fixed.

The D-SPECT system has been shown at several trade shows and has recently obtained Food and Drug Administration approval. It has a significantly different design consisting of 10 individual collimated pixelated CZT modules, as shown in Figure 3B. The patient reclines on a chair with the right-angle gantry positioned over the chest. After a fast scout scan is performed to determine the location of the heart, the CZT detector modules independently rock back and forth to acquire the heart projection data. As Figure 3B indicates, each detector confines its sampling to the region of the heart. No gantry rotation is necessary. This system is reported to have substantially higher count sensitivity than a conventional SPECT system with a 2-fold improvement in spatial resolution (28).

\section{SPECT/CT}

The concept of combining of a SPECT and CT system was first investigated by Hasegawa et al. (29), but the recent introduction of commercial SPECT/CT systems owes much to the success of PET/CT. What advantages does SPECT/ CT have? First, there is improved attenuation correction, resulting from the more accurate and precise attenuation map that is associated with CT. Second, there is value added to SPECT studies with coregistered anatomic images (30). A combined system ensures efficient access to both image sets and allows the operators better control of patient orientation. A third advantage is the ability to perform complementary diagnostic studies in the same setting, making both of these studies more efficient and convenient for the patient. Challenges associated with SPECT/CT include the increased cost of the equipment and room preparation, patient motion, and CT metal artifacts $(31,32)$. Table 3 summarizes the systems that are currently available, and Figure 4 shows example photographs of these products along with a clinical example.

The Infinia Hawkeye features a dual-detector SPECT system with a slip-ring gantry. A low-power fixed anode $\mathrm{x}$-ray tube is mounted directly to the gantry with the CT detectors mounted directly opposite. The current version of this system has 4 rows of detectors. yielding an axial slice thickness of $5 \mathrm{~mm}$. The in-plane resolution is approximately $2.5 \mathrm{~mm}$ (1.2-mm pixel size). In this configuration it takes about $5 \mathrm{~min}$ to scan the $40 \mathrm{~cm}$ corresponding to the SPECT axial field of view. Although the CT images are not equivalent to those produced by dedicated CT scanners, they are far superior to the tomographic images produced by any of the radionuclide transmission products. The $\mathrm{x}$-ray
FIGURE 3. New cardiac SPECT systems. (A) CardiArc uses slit-slat approach to acquire projection information. (B) D-SPECT detector head consists of 10 pixelated CZT detector columns. After a scout scan determines heart location, each detector independently swivels to collect projections.

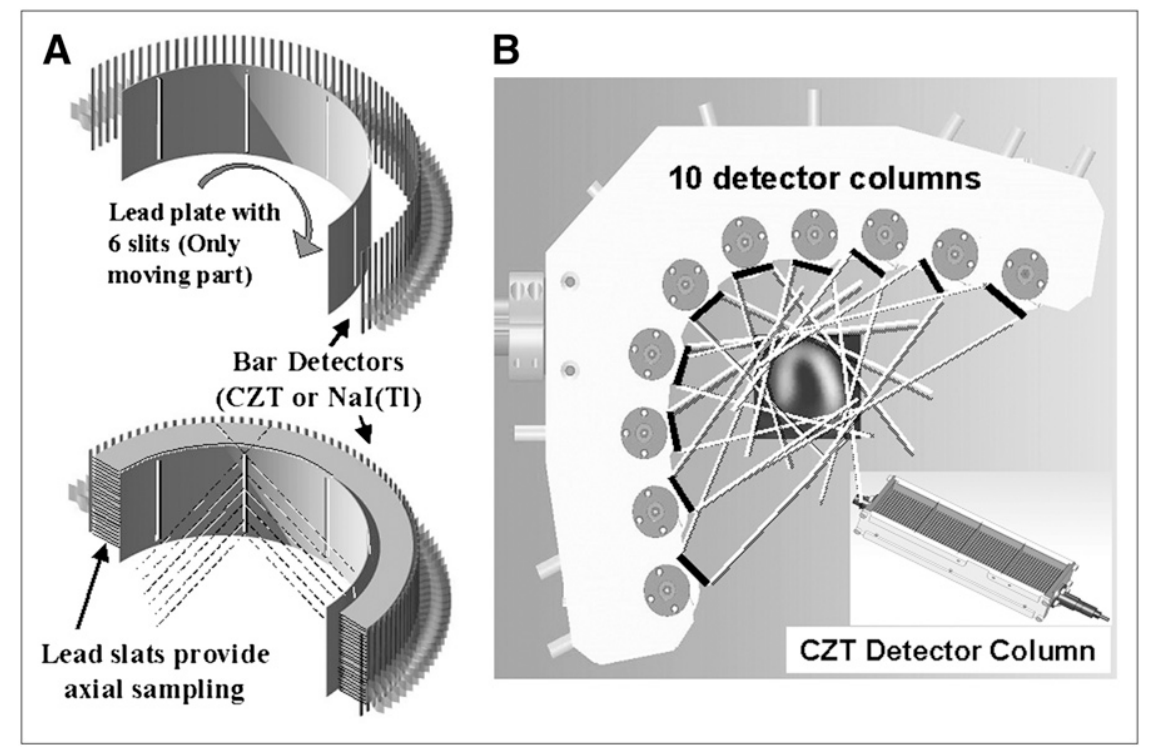


TABLE 3

Commercially Available SPECT/CT Systems

\begin{tabular}{|c|c|c|c|}
\hline & Infinia Hawkeye & Precedence & True Point \\
\hline Manufacturer & GE Healthcare & Philips & Siemens \\
\hline SPECT System & Infinia & Skylight & Symbia \\
\hline CT system & Hawkeye & Brilliance & $\mathrm{T}, \mathrm{T} 2, \mathrm{~T} 6$ \\
\hline No. of CT slices & 1 or 4 & 6 or 16 & 2 or 6 \\
\hline Slice thickness (mm) & 10 or 5 & $0.6-12$ & $0.6-10^{*}$ \\
\hline Tube rotation (s) & 23 & 0.5 & $0.6-1.5^{\star}$ \\
\hline Standard HC resolution (Ip/cm) (2\% MTF) & $>3$ & 13 & $15^{\star}$ \\
\hline Room dimensions $(\mathrm{cm})$ & $419 \times 470$ & $711 \times 442$ & $640 \times 358$ \\
\hline
\end{tabular}

exposure from the Hawkeye is very low and generally requires minimal additional room shielding.

The Philips Precedence combines the "gantry-less" Skylight SPECT system with a Brilliance CT scanner. In its stand-alone version, the Skylight detectors are suspended from moving arms mounted on the ceiling of the room. In the Precedence, the support mechanism for the Skylight is mounted as a bonnet on top of the CT gantry. The Precedence is available in 6- and 16-slice models and produces diagnosticquality CT images. CT slice thickness can be adjusted down to $0.65 \mathrm{~mm}$ and whole-body CT studies can be acquired in $<60$ s. Additional room shielding is required for the Precedence and the size of the room to house this device is larger than that of a conventional SPECT system.

The Siemens True Point combines an Emotion CT scanner with the Symbia SPECT system in a common gantry housing. The system can be purchased with the capability of only doing attenuation correction (Symbia T) or with 2- or 6-slice diagnostic CT capability. On the Symbia T6, CT slice thicknesses down to $0.63 \mathrm{~mm}$ are available, whereas the minimum slice thickness of the Symbia T2 is $1 \mathrm{~mm}$. The Symbia T is operated in a low-dose mode and the slice thickness matches that of the SPECT study. The spatial resolution in this mode is 4.3 line pairs per centimeter $(\mathrm{lp} / \mathrm{cm})$, substantially lower than the 14.1 $\mathrm{lp} / \mathrm{cm}$ available from the Symbia T2. Like the Precedence, the True Point requires a larger room than a conventional SPECT system, and additional room shielding is required for the Symbia T2 and T6 models.

\section{Collimation}

With the exception of the CardiArc, D-SPECT, and MarCSPECT, the devices described earlier acquire SPECT studies in a conventional manner that is not substantially different from the early 1980s when SPECT systems were first introduced. Nearly all clinical SPECT uses parallel-hole collimation as the image-forming aperture, although fanbeam collimation is used for brain SPECT in some clinics. Collimators are very inefficient allowing, $<1$ of 5,000 $\gamma$-rays that hit its surface to be transmitted through to the detector, and are the limiting component of count sensitivity and spatial resolution. In this section, alternate approaches to parallelhole collimation will be discussed. As is often the case, several of these approaches are based on previous ideas that were ahead of the available technology of their time.

The very first collimator used by Anger in the development of the scintillation camera was a pinhole collimator. In the

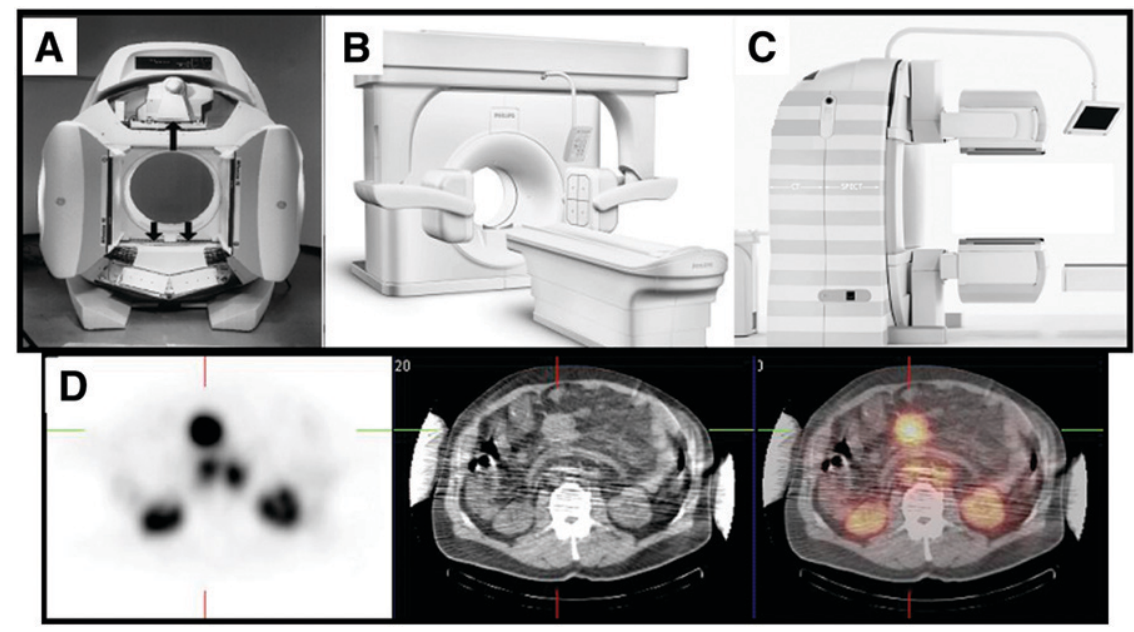

FIGURE 4. SPECT/CT systems. (A) GE Healthcare Infinia Hawkeye. (B) Philips Precedence. (C) Siemens Symbia True Point. (D) Example of clinical ${ }^{111}$ In-pentetreotide SPECT/CT study of patient with carcinoid. 
past $5 \mathrm{y}$ there has been a renewed interest in pinhole imaging driven primarily by small-animal SPECT. Although pinhole imaging has low count sensitivity for imaging large distributed sources, it is very attractive for imaging localized distributions. Through magnification, pinhole imaging reduces the apparent intrinsic spatial resolution of the detector, resulting in an overall spatial resolution that is predominantly determined by the hole size. The accurate modeling of the pinhole aperture has become an important factor in the design of the collimators and the reconstruction algorithms. As a result, there have been several articles published in the recent past reanalyzing the physics of pinhole imaging (33-38).

Although spectacular spatial resolution can be achieved through magnification with pinhole collimation, it comes with the very real price of either very large detectors or limited field of view. The need for magnification is reduced for detectors with good intrinsic spatial resolution. Though most of the currently available detectors have intrinsic spatial resolution in the range of $2.5-3.5 \mathrm{~mm}$, it is possible to achieve performance that is nearly an order of magnitude better by using very small pixelated detectors. The SemiSPECT device described by Kim et al. (21) uses CZT with an intrinsic resolution of $0.380 \mathrm{~mm}$, and a small-animal imager described by Funk et al. (17) has an intrinsic resolution of $0.5 \mathrm{~mm}$.

The count sensitivity of pinhole SPECT systems can be improved by adding pinholes. When using a conventional reconstruction algorithm, the best performance is achieved when there is minimal overlapping of the images projected onto the detector surface. For small-animal SPECT systems that image the entire mouse or rat simultaneously, this limits the number of pinholes to approximately 10 per detector. However, if the imaging volume is constrained to a smaller region, a much larger number of pinholes can be accommodated.

Several other approaches are being resurrected from the early days of nuclear medicine including coded apertures (39) and the Compton $\gamma$-camera (40). Another method for replacing conventional collimation was investigated by Zeng and Gagnon (41). Their device consisted of a linear, positionsensitive CZT detector collimated with a set of parallel lead slats. To collect the projections required for SPECT, the device spins about its central axis as it revolves around the object. In all 3 of these approaches, there is a significant gain in count sensitivity but, in each case, there is also a decoding penalty that exists from the extreme overlapping of the acquired projections. They all perform very well for point source distributions, but their advantage over conventional collimation decreases rather rapidly as the source size increases.

\section{SMALL-ANIMAL SPECT}

The development of new radiopharmaceuticals is crucial to the future of nuclear medicine, and a necessary step in evaluating these products is animal studies that are most often performed in mice or rats. Even more importantly, transgentic and knockout mice are now widely used in medical research to investigate the molecular mechanisms of disease (42-44). Because of their ability to detect very low (picomolar) concentrations of tracer materials, both PET and SPECT are very attractive modalities for molecular imaging in these animal models. Much of the research in this area is being done in the PET arena because of the high count sensitivity of coincidence detection and because of the enormous range of tracers that can be labeled with ${ }^{11} \mathrm{C}$ and ${ }^{18} \mathrm{~F}$. However, single-photon radionuclides have some advantages that are not available with positron emitters. These include a wide range of ${ }^{99 \mathrm{~m}} \mathrm{Tc},{ }^{123} \mathrm{I}$, and ${ }^{111} \mathrm{In}$ tracers developed for human studies, longer physical halflives that better match the biologic kinetics of the radiolabeled agents, and the ability to acquire simultaneous multiple energy studies.

On the basis of the performance of clinical SPECT scanners, one might be pessimistic about the utility of SPECT for imaging small animals. However, when the required field of view is small and the sources are also confined, very high resolution SPECT is possible $(4,8,45,46)$. Spatial resolution below 2-mm full width at half maximum is routinely achieved with investigational and commercial systems, and submillimeter resolution can be achieved by concentrating on small source distributions within the animal. The biggest challenge for small-animal SPECT is the detection efficiency, which is often $<0.1 \%$.

The investigational small animal SPECT systems involve a wide range of instrumentation. Many of the devices use retired clinical SPECT systems that have been fitted with one or more high-resolution pinholes. Others devices have been designed around multiple small-field-of-view $\gamma$-cameras with good intrinsic spatial resolution. These devices also use a wide variety of detection instrumentation, including conventional scintillation cameras, pixelated detectors with PSPMTs or APDs, and semiconductor $\gamma$-cameras. A brief summary of several of the systems is given below to illustrate the approaches that are being used for small-animal SPECT.

Several of the investigational systems are based on clinical triple-detector SPECT systems. The system described by Metzler et al. uses high-resolution pinhole collimation with conventional rotation of the detectors around the animal (47). In addition to the usual concerns about uniformity and center of rotation, motion of the pinhole apertures due to mechanical sag and flexion as the gantry changes position have to be accurately measured and compensated in the reconstruction algorithm (48). Schramm et al. described a similar device with the addition of 7-10 pinholes for each aperture (49). Although this configuration results in some overlap of the projections from the individual pinholes, the expected increase in count sensitivity was achieved with little loss in spatial resolution.

Still another device based on a clinical triple-detector SPECT system is the U-SPECT introduced by Beekman et al. (50). In this approach, the animal is placed inside a cylinder 


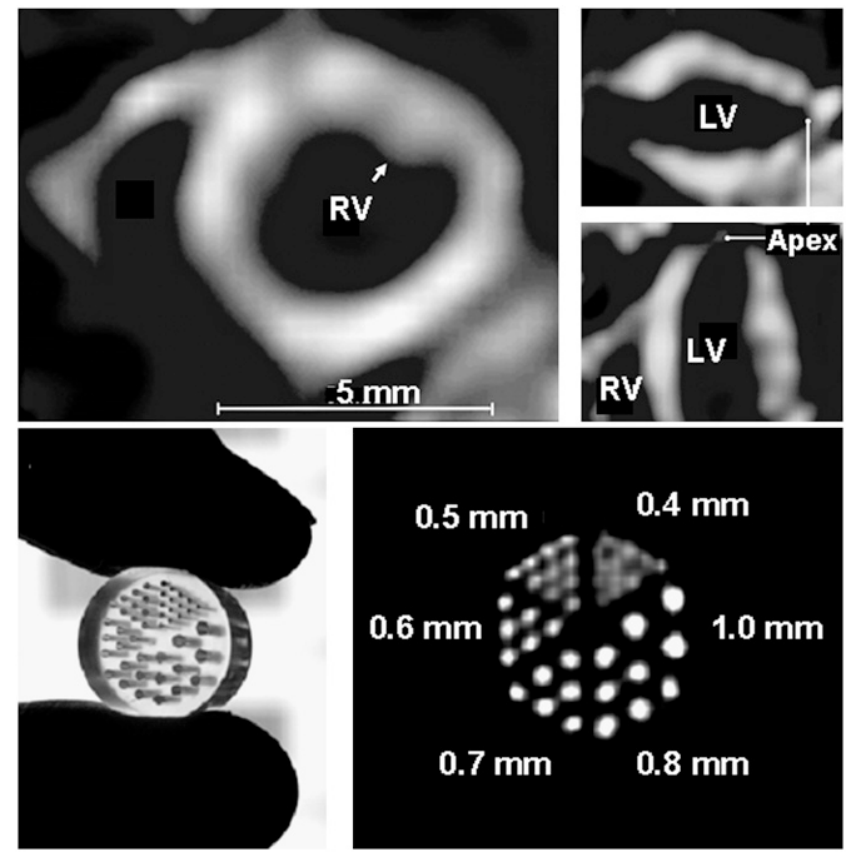

FIGURE 5. Mutual perpendicular cross-sections through submillimeter-resolution 3D myocardial perfusion image volume of living mouse (50). Image data were acquired over $30 \mathrm{~min}$, starting $30 \mathrm{~min}$ after administration of $222 \mathrm{MBq}(6 \mathrm{mCi})$ of $99 \mathrm{mT}$ c-tetrofosmin. On left a short-axis slice shows myocardial perfusion in right ventricular (RV) and left ventricular (LV) walls. Perfusion in anterior papillary muscle (arrow) can be distinguished from other parts of LV wall. On top right is a vertical long-axis slice; on bottom right is a horizontal long-axis slice. Images at bottom show a hot rod phantom and the reconstructed cross-sectional image, with a slice thickness of $0.5 \mathrm{~mm}$.

with 75 pinholes arranged in 5 rings of 15 pinholes. This provides sufficient sampling so that there is no need for either detector or aperture rotation. However, high resolution is achieved at the expense of the field of view, and whole-body mouse imaging requires translation of the animal. Figure 5 shows an example of SPECT images obtained from this device.

The MouseSPECT system developed by the Imaging Physics Center at the National Institutes of Health is based on the CeraSPECT annular $\gamma$-camera (51). The imaging aperture consists of eight 1-mm pinholes that surround the animal. Projections are acquired onto the cylindric surface of the detector as the aperture is rotated about the animal.
The reconstructed spatial resolution of the system is 1.7 $\mathrm{mm}$, with a sensitivity of $373 \mathrm{cps} / \mathrm{MBq}$ at the center of the field of view.

The FastSPECT II system was designed and built at the Center for Gamma Ray Imaging at the University of Arizona to provide dynamic SPECT. The FastSPECT II system has sixteen $12 \times 12 \mathrm{~cm} \mathrm{NaI}(\mathrm{Tl}) \gamma$-cameras arranged in 2 offset rings of 8 cameras each. Event localization within each camera is based on an optimized mapping algorithm that minimizes the edge dead space and provides better performance than Anger logic. A 16-pinhole aperture surrounding the animal provides sufficient projection sampling so that no rotation of the aperture or cameras is required, allowing rapid dynamic SPECT. The detection efficiency of this device is approximately $0.03 \%$ (52).

Another device developed at the Center for Gamma Ray Imaging is the SemiSPECT system, which uses 8 CZT position-sensitive detectors (21). Each detector module has a field of view of $2.7 \times 2.7 \mathrm{~cm}$, and the detector pixel size is $0.33 \mathrm{~mm}$, with a $0.38-\mathrm{mm}$ pitch. In this device, the detectors and the apertures are stationary while the animal is rotated. With $10 \%$ energy windows centered on the ${ }^{99 \mathrm{~m}} \mathrm{Tc}$ photopeak, this system achieves a spatial resolution of 1.4 $\mathrm{mm}$, with an overall system efficiency of $0.05 \%$.

The Linoview system represents a departure from the other systems. The other systems collect sinogram datathat is, the projections are sampled at different angles. In this design, the projections are sampled at different linear locations and arranged as linograms. The 4 pixelated $\mathrm{CsI}(\mathrm{Na})$ detectors that comprise the system each scan across the object with slit apertures generating a rectangular orbit (10). A similar approach has also been described by Lackas et al. (53).

\section{Commercial Small-Animal SPECT Systems}

The advantages of SPECT described for applications with transgenic and knockout mice have been recognized in the marketplace. In the past several years, a large number of small-animal SPECT systems have become commercially available (Table 4), and several of them were developed directly from the research systems described earlier. In addition to producing high-quality, high-resolution SPECT, many of these systems have CT and PET options that operate from a common gantry. The Web site URLs are

TABLE 4

Commercial Small-Animal SPECT Systems

\begin{tabular}{lll}
\hline \multicolumn{1}{c}{ Manufacturer } & \multicolumn{1}{c}{ Device } & \\
\hline Bioscan & NanoSPECT & www.bioscan.com/product.php?p=nanospect \\
Gamma Medica & X-SPECT & www.gammamedica.com/X-SPECT.php \\
GE Healthcare & Explore SPECT-CZT & www.gehealthcare.com/usen/fun_img/pcimaging/index.html \\
Molecular Imaging & U-SPECT & www.milabs.com \\
NeuroPhysics & MollyQ & www.neurophysics.com/products/products.html \\
Siemens & Inveon & www.medical.siemens.com/webapp/wcs/stores/servlet/ProductDisplay? \\
& & storeld=10001\&langld=-11\&catalogld=-11\&catTree =100001,1006503\&productld= \\
& & 16892 \\
\end{tabular}


given so that the interested reader can find additional information.

\section{RECONSTRUCTION AND IMAGE PROCESSING}

Tomographic slices are reconstructed from the acquired projection data using either analytic or iterative algorithms. Analytic reconstructions represent an exact mathematic solution, and there is a general solution for true projection data: filtered backprojection (54). Although filtered backprojection is a relatively efficient operation, it does not always perform well on noisy projections and, as is the case with SPECT data, it generates artifacts when the projections are not line integrals of the internal activity. Several articles have been recently published on analytic solutions for 3D SPECT, but these make assumptions about uniform attenuation and are not general solutions $(55,56)$. However, these algorithms may prove useful in mouse or rat imaging, where overall attenuation is small and deviation from the assumption of uniform attenuation may not be a significant issue.

Iterative algorithms are a preferred alternate method for performing SPECT reconstruction, and over the past $5 \mathrm{y}$ there has been a shift from filtered backprojection to iterative reconstruction in most clinics (57-59). Figure 6 illustrates the basic steps involved in iterative reconstruction. The big advantage of the iterative approach is that accurate corrections can be made for all physical properties of the imaging system and the transport of $\gamma$-rays that can be mathematically modeled. This includes attenuation, scatter, septal penetration, and spatial resolution. In addition, streak artifacts common to filtered backprojection are largely eliminated with iterative algorithms. A major ad-

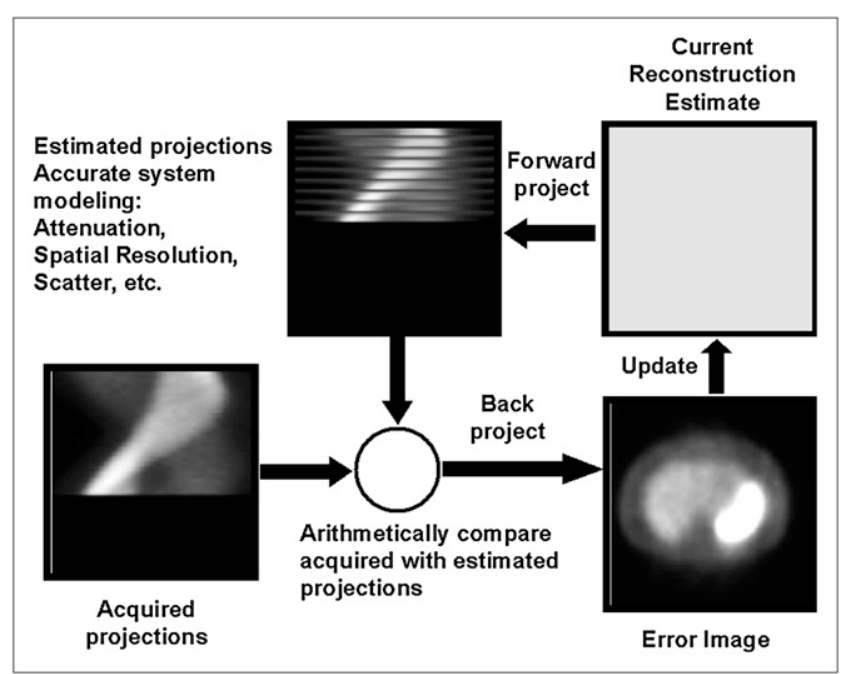

FIGURE 6. Iterative reconstruction. Projections calculated from the estimated distribution are arithmetically compared with measured data and the result backprojected to update the next estimate. Corrections for attenuation, scatter, and spatial resolution are made by mathematically modeling these factors into the forward and backprojection calculations. vance was the introduction of the ordered-subset expectation maximization approach, which produces usable results with a small number of iterations. Much of the developmental work for iterative algorithms was completed before 2000, and there have been few new algorithms introduced since that time. However, important work continues in making the algorithms more efficient.

\section{Dynamic SPECT}

One of the fundamental assumptions given in the introduction is that the distribution of radioactivity does not change during the duration of the scan (i.e., the collection of projection data). This assumption can be modified to include distributions where the activity changes monotonically during acquisitions. When this is the case, it is actually possible to derive additional information about the kinetics of the distribution and to remove the artifacts associated the time inconsistent projections. One technique that has been developed to handle this type of data is referred to as dynamic SPECT (60). With dynamic SPECT, projections are acquired with the usual relatively slow rotation of a conventional SPECT study. If the activity within the volume is either monotonically increasing or decreasing, this behavior can be modeled into the reconstruction algorithm to yield sequential time-sampled tomographic images. This approach requires multiple detectors (at least 2, and 3 are better) and a customized reconstruction algorithm. Although not widely available, dynamic SPECT has been shown to be effective in reducing artifacts resulting from increasing bladder activity in bone SPECT of the pelvis (61). It has also been shown to be valuable in dynamic gated cardiac studies (62).

\section{Corrections}

The point has been made several times in this article that the information acquired by SPECT systems does not represent the projection information required for accurate reconstruction. Although attenuation is the primary factor that makes the projections inconsistent, scattered radiation, spatial resolution, and noise all combine to degrade the quality of the tomographic slices. Efforts to compensate for these problems are rewarded with improved image quality and the capability to quantify activity concentrations $(27,63)$. Much of the work in this area was performed over the past decade, but new techniques continue to evolve with the increased computer capacity and computational speed. For example, scatter correction in myocardial perfusion imaging is accomplished by subtracting counts acquired in off-peak energy windows. This approach is computationally simple but increases image noise. Model-based scatter correction provides a $10 \%-20 \%$ improvement in contrast over the subtraction techniques and can be implemented on clinical computers (64). Other areas where sophisticated SPECT corrections are being applied include brain SPECT and dosimetric studies $(65,66)$.

Another area where considerable progress has been made is in the quantitative evaluation of SPECT image quality. It 
has long been realized that the performance of an imaging system must be considered in the context of a specific task and that an observer study with receiver-operatingcharacteristic analysis is required for scientifically valid

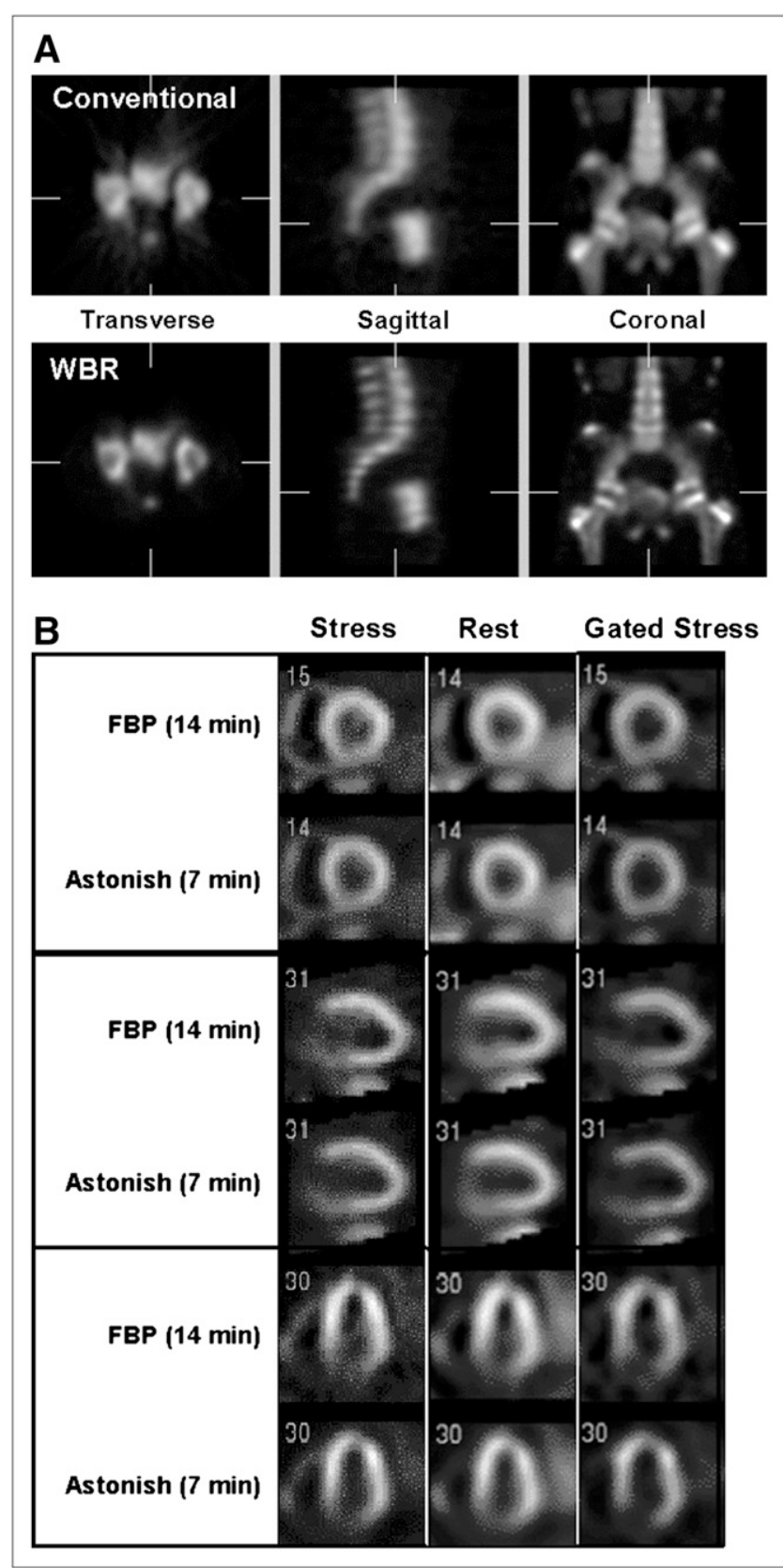

FIGURE 7. Example images from new image-processing algorithms. (A) Wide Beam Reconstruction (WBR) applied to bone SPECT. The top row shows the conventional SPECT views, whereas the bottom row shows the results when WBR is applied to the same patient data. (Images courtesy of Vanderbilt University Medical Center, Nashville, TN.) (B) Astonish method applied to myocardial perfusion SPECT. The stress, rest, and gated components of a ${ }^{99 m T c-M I B I}$ myocardial SPECT study processed with filtered backprojection are compared with Astonish processed data acquired in half the time. (Images courtesy of Radiologic Associates of Sacramento.) conclusions. Because studies with human observers are very expensive and time-consuming, considerable effort within the SPECT field has been devoted to the development of numeric observers that are predictive of human behavior (67-71).

\section{Commercial Image-Processing Software}

The translation of image-processing techniques from the research arena to the clinic has been occurring, and several new commercial products for improving the quality of SPECT and planar images have recently become available. Examples of these products include Astonish from Philips, Evolution from GE Healthcare (69), Wide Beam Reconstruction from UltraSPECT (72), and Flash 3D from Siemens (73). These products incorporate sophisticated corrections for scatter and spatial resolution along with noise suppression (Fig. 7). This has provided improved spatial resolution with an increased signal-to-noise ratio. Proponents of these methods are using the improved image quality to reduce the acquisition time for myocardial perfusion and other SPECT studies by a factor of 2 . It should be noted that the gain in image performance from these algorithms is likely to be dependent on the specific imaging task. The substantial improvement in spatial resolution observed from line or point source images is not expected to be realized for larger more complicated distributions. However, there appears to a real gain in using these processing products, and investigations of their efficacy are ongoing.

\section{CONCLUSION}

SPECT continues to progress with major improvements in instrumentation and reconstruction. The limits of spatial resolution and sensitivity have not yet been reached either for clinical devices or for small-animal imagers. Improvements in detector technology and image-forming apertures will yield intrinsically better projection information. At the same time, refinements in corrections for scattered radiation, attenuation, and spatial resolution losses will result in more accurate and quantitative reconstructed tomographic slices. The capability for combining this information with correlative imaging, both anatomic and functional, from other modalities is also expected to improve.

\section{REFERENCES}

1. Jaszczak RJ. The early years of single photon emission computed tomography (SPECT): an anthology of selected reminiscences. Phys Med Biol. 2006;51:R99R115.

2. Wernick MN, Aarsvold JN, eds. Emission Tomography: The Fundamentals of SPECT and PET. 1st ed. San Diego, CA: Elsevier; 2004.

3. Levin CS. Application-specific small field of view nuclear emission imagers in medicine. In: Wernick MN, Aarsvold JN, eds. Emission Tomography: The Fundamentals of SPECT and PET. 1st ed. San Diego, CA: Elsevier; 2004: 293-334.

4. Levin CS. Primer on molecular imaging technology. Eur J Nucl Med Mol Imaging. 2005;32(suppl 2):S325-S345.

5. Zaidi H. Recent developments and future trends in nuclear medicine instrumentation. Z Med Phys. 2006;16:5-17. 
6. Melcher CL. Perspectives on the future development of new scintillators. Nucl Instrum Methods Phys Res A. 2005;537:6-14.

7. Wilkinson F. Scintillators. In: Wernick MN, Aarsvold JN, eds. Emission Tomography: The Fundamentals of SPECT and PET. 1st ed. San Diego, CA: Elsevier; 2004:229-254.

8. Meikle SR, Kench P, Kassiou M, Banati RB. Small animal SPECT and its place in the matrix of molecular imaging technologies. Phys Med Biol. 2005;50: R45-R61.

9. Wong TZ, Turkington TG, Polascik TJ, Coleman RE. ProstaScint (capromab pendetide) imaging using hybrid gamma camera-CT technology. AJR. 2005; 184:676-680.

10. Walrand S, Jamar F, de Jong M, Pauwels S. Evaluation of novel whole-body high-resolution rodent SPECT (Linoview) based on direct acquisition of linogram projections. J Nucl Med. 2005;46:1872-1880.

11. Moses WW, Shah KS. Potential for RbGd2Br7:Ce, LaBr3:Ce, LaBr3:Ce, and LuI3:Ce in nuclear medical imaging. Nucl Instrum Methods Phys Res A. 2005; 537:317-320.

12. Hammond W, Tekabe Y, Johnson L, et al. Development of high performance mini gamma cameras based on LaBr3 scintillator and H8500 and H9500 PSPMTs and their use in small animal studies. Paper presented at: 2006 IEEE Medical Imaging Conference, October 29-November 5, 2006; San Diego, CA.

13. Pichler BJ, Ziegler SI. Photodetectors. In: Wernick MN, Aarsvold JN, eds. Emission Tomography: The Fundamentals of SPECT and PET. 1st ed. San Diego, CA: Elsevier; 2004:255-269.

14. Loudos GK, Nikita KS, Uzunoglu NK, et al. Improving spatial resolution in SPECT with the combination of PSPMT based detector and iterative reconstruction algorithms. Comput Med Imaging Graph. 2003;27:307-313.

15. Pani R, Pellegrini R, Cinti MN, et al. New devices for imaging in nuclear medicine. Cancer Biother Radiopharm. 2004;19:121-128.

16. Zeniya $\mathrm{T}$, Watabe $\mathrm{H}$, Aoi $\mathrm{T}$, et al. Use of a compact pixellated gamma camera for small animal pinhole SPECT imaging. Ann Nucl Med. 2006;20:409-416.

17. Funk T, Despres P, Barber WC, Shah KS, Hasegawa BH. A multipinhole small animal SPECT system with submillimeter spatial resolution. Med Phys. 2006;33:1259-1268.

18. Moehrs S, Del Guerra A, Herbert DJ, Mandelkern MA. A detector head design for small-animal PET with silicon photomultipliers (SiPM). Phys Med Biol. 2006;51:1113-1127.

19. Darambara DG, Todd-Pokropek A. Solid state detectors in nuclear medicine. $Q J$ Nucl Med. 2002;46:3-7.

20. Wagenaar DJ. CdTe and CdZnTe semiconductor detectors for nuclear medicine imaging. In: Wernick MN, Aarsvold JN, eds. Emission Tomography: The Fundamentals of SPECT and PET. 1st ed. San Diego, CA: Elsevier; 2004:270292.

21. Kim H, Furenlid LR, Crawford MJ, et al. SemiSPECT: a small-animal singlephoton emission computed tomography (SPECT) imager based on eight cadmium zinc telluride (CZT) detector arrays. Med Phys. 2006;33:465-474.

22. Rogers WL, Clinthorne NH, Bolozdynya A. Compton camera for nuclear medical imaging. In: Wernick MN, Aarsvold JN, eds. Emission Tomography: The Fundamentals of SPECT and PET. 1st ed. San Diego, CA: Elsevier; 2004:383-420.

23. Nagarkar VV, Shestakova I, Gaysinskiy V, et al. A CCD-based detector for SPECT. Paper presented at: IEEE Nuclear Science Symposium, October 16-22, 2004; Rome, Italy.

24. Beekman FJ, de Vree GA. Photon-counting versus an integrating CCD-based gamma camera: important consequences for spatial resolution. Phys Med Biol. 2005;50:N109-N119.

25. Bateman TM, Cullom SJ. Attenuation correction single-photon emission computed tomography myocardial perfusion imaging. Semin Nucl Med. 2005;35: 37-51.

26. Zaidi H, Hasegawa B. Determination of the attenuation map in emission tomography. J Nucl Med. 2003;44:291-315.

27. Zaidi H, Koral KF. Scatter modelling and compensation in emission tomography. Eur J Nucl Med Mol Imaging. 2004;31:761-782.

28. Patton JA, Sandler MP, Berman D. D-SPECT: a new solid state camera for high speed molecular imaging [abstract]. J Nucl Med. 2006;47(suppl 1):189P.

29. Hasegawa BH, Iwata K, Wong KH, et al. Dual-modality imaging of function and physiology. Acad Radiol. 2002;9:1305-1321.

30. Schillaci O. Hybrid SPECT/CT: a new era for SPECT imaging? Eur J Nucl Med Mol Imaging. 2005;32:521-524.

31. Hasegawa BH, Wong KH, Iwata K, et al. Dual-modality imaging of cancer with SPECT/CT. Technol Cancer Res Treat. 2002;1:449-458.

32. O'Connor MK, Kemp BJ. Single-photon emission computed tomography/ computed tomography: basic instrumentation and innovations. Semin Nucl Med. $2006 ; 36: 258-266$.
33. Accorsi R, Metzler SD. Analytic determination of the resolution-equivalent effective diameter of a pinhole collimator. IEEE Trans Med Imaging. 2004;23:750-763.

34. Accorsi R, Metzler SD. Resolution-effective diameters for asymmetric-knifeedge pinhole collimators. IEEE Trans Med Imaging. 2005;24:1637-1646.

35. Beque D, Nuyts J, Bormans G, Suetens P, Dupont P. Characterization of pinhole SPECT acquisition geometry. IEEE Trans Med Imaging. 2003;22:599-612.

36. Hsu CH, Huang PC. A geometric system model of finite aperture in small animal pinhole SPECT imaging. Comput Med Imaging Graph. 2006;30:181-185.

37. Israel-Jost V, Choquet P, Salmon S, Blondet C, Sonnendrucker E, Constantinesco A. Pinhole SPECT imaging: compact projection/backprojection operator for efficient algebraic reconstruction. IEEE Trans Med Imaging. 2006;25:158-167.

38. Metzler SD, Accorsi R. Resolution- versus sensitivity-effective diameter in pinhole collimation: experimental verification. Phys Med Biol. 2005;50:5005-5017.

39. Mu Z, Liu YH. Aperture collimation correction and maximum-likelihood image reconstruction for near-field coded aperture imaging of single photon emission computerized tomography. IEEE Trans Med Imaging. 2006;25:701-711.

40. Singh M, Doria D. An electronically collimated gamma camera for single photon emission computed tomography. Part II. Image reconstruction and preliminary experimental measurements. Med Phys. 1983;10:428-435.

41. Zeng GL, Gagnon D. Image reconstruction algorithm for a spinning strip CZT SPECT camera with a parallel slat collimator and small pixels. Med Phys. 2004; 31:3461-3473.

42. Davey RA, MacLean HE. Current and future approaches using genetically modified mice in endocrine research. Am J Physiol Endocrinol Metab. 2006;291:E429-E438.

43. Bolon B. Genetically engineered animals in drug discovery and development: a maturing resource for toxicologic research. Basic Clin Pharmacol Toxicol. 2004; 95:154-161.

44. Kimura S, Gonzalez FJ. Applications of genetically manipulated mice in pharmacogenetics and pharmacogenomics. Pharmacology. 2000;61:147-153.

45. Chatziioannou AF. Instrumentation for molecular imaging in preclinical research: micro-PET and micro-SPECT. Proc Am Thorac Soc. 2005;2: 510-511, 533-536.

46. Peremans K, Cornelissen B, Van Den Bossche B, Audenaert K, Van de Wiele C. A review of small animal imaging planar and pinhole SPECT gamma camera imaging. Vet Radiol Ultrasound. 2005;46:162-170.

47. Metzler SD, Jaszczak RJ, Patil NH, Vemulapalli S, Akabani G, Chin BB. Molecular imaging of small animals with a triple-head SPECT system using pinhole collimation. IEEE Trans Med Imaging. 2005;24:853-862.

48. Metzler SD, Greer KL, Jaszczak RJ. Determination of mechanical and electronic shifts for pinhole SPECT using a single point source. IEEE Trans Med Imaging. 2005;24:361-370.

49. Schramm NU, Ebel G, Engelanf U, Schurrat T, Behe M, Behr TM. Highresolution SPECT using multiple pinhole collimation. IEEE Trans Nucl Sci. 2003;50:315-320

50. Beekman FJ, van der Have F, Vastenhouw B, et al. U-SPECT-I: a novel system for submillimeter-resolution tomography with radiolabeled molecules in mice. J Nucl Med. 2005;46:1194-1200.

51. Goertzen AL, Jones DW, Seidel J, Li K, Green MV. First results from the highresolution mouseSPECT annular scintillation camera. IEEE Trans Med Imaging. 2005;24:863-867.

52. Furenlid LR, Wilson DW, Yi-chun C, et al. FastSPECT II: a second generation high-resolution dynamic SPECT imager. IEEE Trans Nucl Sci. 2004;51:631-635.

53. Lackas C, Schramm NU, Hoppin JW, Engeland U, Wirrwar A, Halling H. T-SPECT: a novel imaging technique for small animal research. IEEE Trans Nucl Sci. 2005;52:181-187.

54. Bruyant PP. Analytic and iterative reconstruction algorithms in SPECT. J Nucl Med. 2002;43:1343-1358.

55. Huang Q, Zeng GL, You J, Gullberg GT. An FDK-like cone-beam SPECT reconstruction algorithm for non-uniform attenuated projections acquired using a circular trajectory. Phys Med Biol. 2005;50:2329-2339.

56. Tang Q, Zeng GL, Gullberg GT. Analytical fan-beam and cone-beam reconstruction algorithms with uniform attenuation correction for SPECT. Phys Med Biol. 2005;50:3153-3170.

57. Defrise M, Gullberg GT. Image reconstruction. Phys Med Biol. 2006;51: R139-R154.

58. Lalush DA, Wernick MN. Iterative image reconstruction. In: Wernick MN, Aarsvold JN, eds. Emission Tomography: The Fundamentals of SPECT and PET. 1st ed. San Diego, CA. Elsevier; 2004:443-472.

59. Qi J, Leahy RM. Iterative reconstruction techniques in emission computed tomography. Phys Med Biol. 2006;51:R541-R578.

60. Celler A, Farncombe T, Bever C, et al. Performance of the dynamic single photon emission computed tomography (dSPECT) method for decreasing or increasing activity changes. Phys Med Biol. 2000;45:3525-3543.

61. Wells RG, Farncombe T, Chang E, Nicholson RL. Reducing bladder artifacts in clinical pelvic SPECT images. J Nucl Med. 2004;45:1309-1314. 
62. Feng B, Pretorius PH, Farncombe TH, et al. Simultaneous assessment of cardiac perfusion and function using 5-dimensional imaging with Tc-99m teboroxime. J Nucl Cardiol. 2006;13:354-361.

63. King MA, Glick SJ, Pretorius PH, et al. Attenuation, scatter and spatial resolution compensation in SPECT. In: Wernick MN, Aarsvold JN, eds. Emission Tomography: The Fundamentals of SPECT and PET. 1st ed. San Diego, CA: Elsevier; 2004:473-498.

64. Xiao J, de Wit TC, Staelens SG, Beekman FJ. Evaluation of 3D Monte Carlobased scatter correction for ${ }^{99 \mathrm{~m}} \mathrm{Tc}$ cardiac perfusion SPECT. J Nucl Med. 2006; 47:1662-1669.

65. Dewaraja YK, Wilderman SJ, Ljungberg M, Koral KF, Zasadny K, Kaminiski MS. Accurate dosimetry in ${ }^{131} \mathrm{I}$ radionuclide therapy using patient-specific, 3-dimensional methods for SPECT reconstruction and absorbed dose calculation. J Nucl Med. 2005;46:840-849.

66. He B, Frey EC. Comparison of conventional, model-based quantitative planar, and quantitative SPECT image processing methods for organ activity estimation using In-111 agents. Phys Med Biol. 2006;51:3967-3981.

67. Barrett HH, Abbey CK, Clarkson E. Objective assessment of image quality. III. ROC metrics, ideal observers, and likelihood-generating functions. J Opt Soc Am A Opt Image Sci Vis. 1998;15:1520-1535.
68. Farncombe TH, Gifford HC, Narayanan MV, Pretorius PH, Frey EC, King MA. Assessment of scatter compensation strategies for ${ }^{67} \mathrm{Ga}$ SPECT using numerical observers and human LROC studies. J Nucl Med. 2004;45:802-812.

69. Frey EC, Gilland KL, Tsui BM. Application of task-based measures of image quality to optimization and evaluation of three-dimensional reconstruction-based compensation methods in myocardial perfusion SPECT. IEEE Trans Med Imaging. 2002;21:1040-1050.

70. Gifford HC, King MA, Pretorius PH, Wells RG. A comparison of human and model observers in multislice LROC studies. IEEE Trans Med Imaging. 2005; 24:160-169.

71. Narayanan MV, King MA, Pretorius PH, et al. Human-observer receiver-operatingcharacteristic evaluation of attenuation, scatter, and resolution compensation strategies for ${ }^{99 m}$ Tc myocardial perfusion imaging. J Nucl Med. 2003;44:1725-1734.

72. Borges-Neto S, Pagnanelli R, Shaw K, Coleman RE. Clinical validation of the wide beam reconstruction method for shortening scan time of gated cardiac SPECT perfusion studies [abstract]. J Nucl Med. 2006;47(suppl 1):272P.

73. Vija H, Hawman EG, Engdahl JC. Analysis of a SPECT OSEM reconstruction method with 3D beam modeling and optional attenuation correction: phantom studies. IEEE Medical Imaging Conference Proceedings, October 16-22, 2004; 2662-2667. 